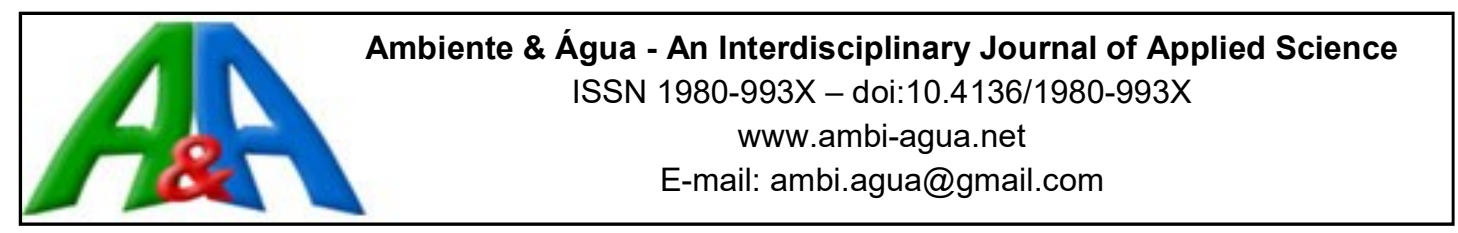

\title{
Vigilância e monitoramento de fluoretos em águas de abastecimento público: uma revisão sistemática
}

\author{
doi:10.4136/ambi-agua.1929 \\ Received: 04 May 2016; Accepted: 31 Aug. 2016 \\ Ceci Queluz Venturini ${ }^{1}$; Paulo Capel Narvai ${ }^{1}$; \\ Marco Antonio Manfredini ${ }^{2}$; Paulo Frazão ${ }^{1^{*}}$ \\ ${ }^{1}$ Universidade de São Paulo (USP/FSP), São Paulo, SP, Brasil \\ Departamento de Prática de Saúde Pública \\ ${ }^{2}$ Centro Colaborador do Ministério da Saúde em Vigilância da Saúde Bucal (USP), São Paulo, SP, Brasil \\ *Autor correspondente: e-mail: pafrazao@usp.br, \\ ceciventurini@gmail.com,pcnarvai@usp.br,manfra@uol.com.br
}

\section{RESUMO}

O fluoreto presente na água pode ser um fator de proteção ou de risco à dentição humana dependendo da sua concentração. O objetivo foi sumarizar as principais características metodológicas e os principais achados descritos em estudos sobre monitoramento da concentração de fluoreto em água de abastecimento público, que foram publicados em revistas científicas especializadas entre 2008 e 2012, examinando suas implicações para a vigilância em saúde pública. Uma revisão sistemática foi realizada buscando artigos nas bases de dados Embase, Lilacs e PubMed. Trinta e seis artigos foram incluídos: 28 das Américas, cinco da Ásia, dois da África e um da Europa. A variabilidade dos estudos quanto ao desenho e aos procedimentos metodológicos foi elevada. Embora tenha sido oferecida informação sobre a distribuição espaço-temporal das amostras, poucos trabalhos descreveram a população coberta pela fonte de abastecimento investigada. Quase metade dos estudos foi do tipo transversal e nenhuma medida de dispersão ou de variação foi associada ao valor médio. Predominou o uso do método eletrométrico e a classificação dicotômica do tipo adequado/inadequado e/ou acima/abaixo para avaliar os resultados. Menos da metade dos estudos esteve articulada à vigilância como ação do Estado, e um número ainda menor levantou hipóteses sobre possíveis fatores associados aos resultados encontrados. Há um espaço importante a ser diminuído entre os resultados dessas iniciativas e o uso das informações pelas autoridades de saúde pública e também para melhorar os procedimentos metodológicos em futuros estudos. A interação entre os pesquisadores e os órgãos de controle da qualidade da água deveria ser incrementada.

Palavras-chave: água potável, flúor, fluoretação da água, saúde pública.

\section{Surveillance and monitoring of fluorides in public drinking water: a systematic review}

\section{ABSTRACT}

Fluoride can harm or protect human dentition according to its concentration in the water. This paper summarizes major methodological features and main findings described in studies 
of fluoride concentration in public supply water that were published in specialized journals between 2008 and 2012, highlighting their implications for public health surveillance. A systematic review was conducted comprising scientific articles in the databases Embase, Lilacs and PubMed. Thirty-six studies were included: 28 from the Americas, five from Asia, two from Africa and one from Europe. The variability of the studies related to design and methodological procedures was high. Although the studies provided information on the spacetemporal distribution of the samples, few publications described the population covered by the investigated supply source. Almost half of the studies were cross-sectional and no dispersion or variation measure was associated to the mean value. The electrometric method and dichotomous classification of the samples were predominately used for evaluating the outcomes. Less than half of the studies were articulated to surveillance as a State action and a lesser number raised hypotheses regarding possible factors related to the findings. There is a major space to be narrowed between the results of these initiatives and the use of that information by public health authorities and to improve methodological procedures in future studies. The interaction between researchers and water quality control organizations should be increased.

Keywords: fluorine, public health, safe water, water fluoridation.

\section{INTRODUÇÃO}

Vigilância em saúde pública pode ser definida como um sistema articulado de ações que asseguram a coleta, análise e interpretação de dados acerca de eventos de saúde específicos que afetam a população, incluindo a rápida disseminação dos resultados para todos aqueles que são responsáveis pela prevenção e controle (Waldman, 1998). A vigilância da água de abastecimento público deve ser parte deste sistema visando assegurar padrões de segurança e qualidade aceitáveis para o consumo humano em conformidade com metas de saúde predeterminadas. A vigilância não substitui ou isenta de responsabilidade empresas e companhias de tratamento da água na realização dos seus controles operacionais (WHO, 2011). Entre os parâmetros físico-químicos e microbiológicos que devem ser objeto de vigilância destaca-se a concentração de fluoreto.

O monitoramento dos teores de fluoreto na água de abastecimento público pode ser realizado apenas com a finalidade de assegurar sua conformidade aos padrões de potabilidade, ou pode também ser empregado para assegurar a qualidade dos teores visando o máximo benefício da prevenção da cárie dentária com o mínimo de risco de fluorose ou manchas no esmalte dentário. Quando esse monitoramento é parte da ação permanente do Estado, ele é chamado de vigilância.

A vigilância pode ser realizada a partir da auditoria de dados produzidos pelas companhias de abastecimento de água, ou por meio da observação direta examinando amostras de água da rede de distribuição (WHO, 2011). Considerando que desfechos decorrentes de medidas preventivas podem ser verificados somente alguns anos após a implementação de tais medidas, especialistas brasileiros têm recomendado que o controle da fluoretação seja feito por organismos não diretamente responsáveis pelo tratamento da água (princípio do heterocontrole), por meio da avaliação direta de amostras de água colhidas na rede de distribuição (Schneider Filho et al. 1992; ENATESPO, 1998; Narvai, 2000b), a fim de assegurar a qualidade do processo, a validade da informação e a confiabilidade para se alcançar as metas de saúde bucal.

Os primeiros sistemas de vigilância dos teores de flúor nas águas de abastecimento público no Brasil foram instituídos no final dos anos 1980, com o objetivo de possibilitar o monitoramento do processo de fluoretação, subsidiando a relação entre o Poder Público e as 
companhias de abastecimento (Schneider Filho et al. 1992; Narvai, 2000b).

A constatação de que em muitas localidades a medida sofria oscilações nas concentrações de fluoreto (Vasconcellos, 1982; Barros et al. 1990; Chan et al., 1993) tem motivado a implantação de sistemas de vigilância sanitária da fluoretação das águas de abastecimento público (Schneider Filho et al. 1992; Narvai, 2000b), e a realização de estudos de monitoramento dos seus teores. Entretanto, nenhuma revisão sistemática dos estudos publicados ou um balanço crítico sobre o assunto foi divulgado. Não se dispõe de informação sobre o desenho dos estudos, as medidas utilizadas, os critérios de avaliação e os principais fatores associados aos resultados encontrados.

O objetivo do presente estudo foi sumarizar as principais características metodológicas e os principais achados descritos em estudos sobre monitoramento da concentração de fluoreto em água de abastecimento público, que foram publicados em revistas científicas especializadas entre 2008 e 2012, examinando suas implicações para vigilância em saúde pública.

\section{MÉTODOS}

Uma revisão sistemática foi empreendida para analisar os estudos de monitoramento da concentração de fluoreto em água de abastecimento público. Revisões sistemáticas representam um método de síntese do conhecimento que requer uma questão claramente formulada, e o uso sistemático e explicito de métodos para identificar, selecionar, extrair e analisar dados de pesquisas relevantes. Elas podem ser usadas para abordar diversas questões de pesquisas, tais como os benefícios e danos decorrentes de um determinado tratamento ou a prevalência de uma determinada condição em um grupo populacional, entre outros aspectos. Tricco et al. (2011) descreveram diferentes tipos de revisão sistemática de acordo com a questão de pesquisa selecionada e os métodos utilizados. Esta revisão consiste de uma síntese narrativa envolvendo uma indagação estruturada e um sumário dos achados dos estudos.

Foram incluídos apenas artigos científicos abordando monitoramento da concentração de fluoreto em água de beber (drinking water) e água de abastecimento (water supply, tap water). Portanto, artigos envolvendo água bruta (raw water) e água subterrânea (groundwater) não foram considerados. Foram incluídos estudos observacionais publicados em inglês, português e espanhol que foram identificados por meio de busca eletrônica no EMBASE, uma base de dados de literatura especializada biomédica, e também nas bases de dados de informação bibliográfica LILACS e PUBMED. As sintaxes das estratégias de busca foram adaptadas para cada base.

Os estudos foram identificados por busca eletrônica realizada em 29/03/2013. As chaves de busca foram: EMBASE \{water sampling AND fluoride\}, \{fluorine AND (filtro) drug (term) AND water (term)\}; LILACS \{flúor (descritor de assunto) AND água (palavra em qualquer campo)\}, \{fluoretação da água (descritor de assunto)\}, \{flúor (palavra em qualquer campo) AND vigilância (palavra em qualquer campo)\}; PUBMED \{"fluorides"[MeSH Terms] OR "fluorides"[All Fields]) AND ("drinking water"[MeSH Terms] OR ("drinking"[All Fields] AND "water"[All Fields]) OR "drinking water"[All Fields]) AND ("epidemiology"[Subheading] OR "epidemiology"[All Fields] OR "surveillance"[All Fields] OR "epidemiology"[MeSH Terms] OR "surveillance"[All Fields]\}, "fluorides" [MeSH Terms] AND "water supply/analysis" [MeSH Terms]\}, \{"fluorides"[MeSH Terms] AND "drinking water" [All fields] AND "monitoring" [All fields]\}.

Um exercício de calibração foi realizado para assegurar confiabilidade na seleção dos resumos antes do processo de busca. Foram apresentadas as características principais de cada base de dados para o primeiro autor. Em seguida, ele realizou exercício de seleção de resumos com base nos primeiros cem registros para cada base de dados. Os achados foram discutidos 
com o último autor. Em caso de dúvida durante o processo de busca, o último autor fez a revisão do registro. Foram considerados os últimos cinco anos a partir da data de busca. Assim, foram incluídos os artigos publicados em revistas científicas especializadas no período de 2008 a 2012.

Uma planilha foi elaborada para a extração dos dados. O primeiro autor executou a extração e o último revisou o preenchimento da planilha e conferiu o conteúdo.

Os artigos incluídos foram organizados segundo o título do estudo, título do periódico, nome do primeiro autor, ano da publicação, objetivo e o conteúdo foi analisado segundo as seguintes categorias lógicas:

a) local do estudo (cidade, país), categorizando os países segundo os continentes;

b) a distribuição espaço-temporal das amostras considerando o período de tempo da coleta de dados; e a presença ou não de diferentes pontos de coleta no território, com a finalidade de caracterizar a extensão espacial e a duração temporal. Estudos com duração de três meses ou menos foram considerados estudos de desenho transversal. Estudos realizados durante um tempo maior foram classificados como tendo desenho longitudinal. Os estudos longitudinais foram agrupados em três intervalos de tempo: 4 a 11 meses; 12 a 59 meses; 60 meses ou mais, sendo utilizada esta divisão devido à exposição do fluoreto na água de consumo humano exigir vários anos para a produção de efeitos sobre a dentição;

c) método de análise da concentração de fluoreto (eletrométrico e colorimétrico do tipo SPANDS e do tipo Alizarina);

d) as medidas utilizadas e o tipo de julgamento adotado para classificar as amostras de água, sendo considerado do tipo dicotômico quando as amostras eram distribuídas em duas categorias (aceitável/inaceitável ou adequado/inadequado); em tricotômico quando as amostras eram distribuídas em três categorias (abaixo do nível ótimo/nível ótimo/acima do nível ótimo) e multinominal quando acima de três categorias, a qual envolve a combinação risco/benefício;

e) critério de julgamento adotado para interpretar os resultados;

f) relação com a vigilância como ação do Estado (sim/não). Foram considerados articulados à vigilância, os estudos que utilizaram dados de concentração de fluoreto em águas de consumo humano produzidos pelas autoridades sanitárias. Estudos que explicitaram a participação de um órgão estatal (instituído e mantido pelo poder público), seja na fase de mapeamento da rede de abastecimento ou na fase de coleta das amostras, também foram considerados, e;

g) possíveis fatores associados aos resultados encontrados.

A qualidade metodológica de cada estudo e o risco de viés não foram avaliados, nem usados como critério de inclusão/exclusão porque um dos objetivos da revisão foi analisar as principais características metodológicas. Uma síntese narrativa foi organizada com base nas categorias lógicas mencionadas. 
Tabela 1. Estudos brasileiros sobre monitoramento da água de abastecimento segundo o primeiro autor, ano de publicação, local, distribuição espacial e temporal das amostras, desenho do estudo e técnica de aferição.

\begin{tabular}{|c|c|c|c|c|c|c|}
\hline $1^{\circ}$ Autor & Ano & Local & Distribuição espacial & $\begin{array}{l}\text { Distribuição } \\
\text { temporal (meses) }\end{array}$ & $\begin{array}{l}\text { Desenho } \\
\text { do estudo }\end{array}$ & $\begin{array}{l}\text { Técnica de } \\
\text { aferição }\end{array}$ \\
\hline Alves et al. & 2008 & Marília-SP & 6 pontos & 2 & Transversal & eletrométrico \\
\hline Catani et al. & 2008 & 10 municípios (8-SP, 1-MG, 1-CE) & critério demográfico, 1 amostra para cada 10 mil hab. & 36 a 132 & Longitudinal & eletrométrico \\
\hline Fernandes-Jr et al. & 2008 & São José dos Campos-SP & 20 residências em cada uma das cinco regiões do municipio & coleta única & Transversal & eletrométrico \\
\hline Panizzi e Peres & 2008 & Chapecó-SC & 10 pontos segundo o porte populacional & 120 & Longitudinal & eletrométrico \\
\hline Scorsafava et al. & 2008 & 29 municípios, Grande São Paulo - SP & não informado & 36 & Transversal & eletrométrico \\
\hline Bellé et al. & 2009 & Campo Grande-MS & 21 pontos em 7 subsdistritos & 60 & Longitudinal & eletrométrico \\
\hline Daré et al. & 2009 & 40 municípios da região de Araçatuba-SP & $\begin{array}{l}\text { amostras em pontos aleatórios buscando-se representar todo o } \\
\text { sistema de distribuição }\end{array}$ & 60 & Longitudinal & eletrométrico \\
\hline Moraes et al. & 2009 & 6 municípios da região Centro-Sul do Paraná & varios endereços - creches e escolas do perímetro urbano & 3 & Transversal & SPADNS \\
\hline Saliba et al. & 2009 & 8 municípios, região Noroeste do estado de SP & $\begin{array}{l}3 \text { pontos por cidade - cada uma é servida por uma unidade de } \\
\text { tratamento }\end{array}$ & 36 & Longitudinal & eletrométrico \\
\hline Silva et al. & 2009 & 164 municípios do Piauí & $\begin{array}{l}\text { duas amostras por cidade, uma da fonte de abastecimento público e } \\
\text { uma da torneira de água residencial ou publica da mesma fonte }\end{array}$ & coleta única & Transversal & eletrométrico \\
\hline Carmo et al. & 2010 & São Luís-MA & pontos definidos segundo os bairros abastecidos & coleta única & Transversal & eletrométrico \\
\hline Leivas et al. & 2010 & Canoas-RS & 22 pontos para as três unidades de tratamento & 8 & Longitudinal & eletrométrico \\
\hline Marmolejo et al. & 2010 & Niterói-RJ & 11 pontos abrangendo 48 bairros e 12 regiões administrativas & 5 & Longitudinal & eletrométrico \\
\hline Sampaio et al. & 2010 & 167 municípios da Paraíba & $\begin{array}{l}3 \text { amostras por cidade; uma da fonte de abastecimento público e duas } \\
\text { proveniente da torneira de agua residencial ou pública da mesma } \\
\text { fonte }\end{array}$ & 9 & Longitudinal & eletrométrico \\
\hline Castro et al.* & 2011 & Campo Alegre de Lourdes-BA & pontos em todos os bairros do município & 2 & Transversal & eletrométrico \\
\hline Cesa et al. & 2011 & capitais brasileiras & não se aplica & 12 & Longitudinal & não se aplica \\
\hline Motter et al. & 2011 & Curitiba-PR & diferentes pontos da cidade & 102 & Longitudinal & $\begin{array}{l}\text { eletrométrico, } \\
\text { SPADNS }\end{array}$ \\
\hline Olivati et al. & 2011 & Capão Bonito, SP & 10 pontos abrangendo as duas principais unidades de tratamento & 12 & Longitudinal & eletrométrico \\
\hline Scorsafava et al. & 2011 & 29 municípios da Grande São Paulo-SP & $\begin{array}{l}\text { critério de densidade populacional, locais estratégicos, de baixa } \\
\text { pressão do sistema, e dist espacial doenças hídricas }\end{array}$ & 36 & Longitudinal & eletrométrico \\
\hline Silva et al. & 2011 & Município da Centro-Oeste-SP & não informado & 60 & Longitudinal & eletrométrico \\
\hline Moimaz et al & 2012 & Araçatuba-SP & 3 pontos para cada fonte de abastecimento/unidade de tratamento & 90 & Longitudinal & eletrométrico \\
\hline Moimaz et al & 2012 & Araçatuba-SP & 3 pontos para cada fonte de abastecimento/unidade de tratamento & 72 & Longitudinal & eletrométrico \\
\hline Moimaz et al. & 2012 & 29 municípios, região Noroeste do estado de SP & 3 pontos para cada fonte de abastecimento/unidade de tratamento & 48 & Longitudinal & eletrométrico \\
\hline Peixoto et al. & 2012 & Jaguaribara-CE & $\begin{array}{l}3 \text { pontos por unidade de tratamento, minimo de cinco p/ municipio } \\
\text { com até } 50 \text { mil hab. }\end{array}$ & 12 & Longitudinal & eletrométrico \\
\hline
\end{tabular}

*Nota: também examinou água engarrafada. 
Tabela 2. Estudos não brasileiros sobre monitoramento da água de abastecimento segundo primeiro autor, ano de publicação, local, distribuição espacial e temporal das amostras, desenho do estudo e técnica de aferição.

\begin{tabular}{|c|c|c|c|c|c|c|}
\hline $1^{\circ}$ Autor & Ano & Local (cidade/país) & Distribuição espacial & $\begin{array}{l}\text { Distribuição } \\
\text { temporal (meses) }\end{array}$ & Desenho do estudo & $\begin{array}{l}\text { Técnica de } \\
\text { aferição }\end{array}$ \\
\hline D'Alessandro et al. & 2008 & 251 cidades na Sicília, Itália & $\begin{array}{l}1 \text { amostra pra cada } 5 \text { mil hab; número + } \\
\text { reduzido para cidades maiores }\end{array}$ & coleta única & Transversal & eletrométrico \\
\hline Poureslami et al.** & 2008 & Koohbanan, Irã & não informado & não informado & Transversal & eletrométrico \\
\hline Akpata et al. & 2009 & Nigéria & 109 áreas do país & coleta única & Transversal & colorimétrico $^{* * *}$ \\
\hline Viswanathan & 2009 & $\begin{array}{l}\text { Nilakottai, Distrito de Dindigul, Tamil Nadu, } \\
\text { India }\end{array}$ & $\begin{array}{l}5 \text { amostras de cada vila (oeste, leste, norte, } \\
\text { sul e centro) }\end{array}$ & não informado & Transversal & eletrométrico \\
\hline Yarmolinsky et al. & 2009 & 34 municípios, Provincia de Ontario, Canadá & não se aplica & 12 & Longitudinal & não se aplica \\
\hline Quock \& Chan & 2010 & Houston, Texas, Estados Unidos & um ponto de coleta & 12 & Longitudinal & eletrométrico \\
\hline Chacón et el. & 2011 & Tlahuac, Cidade do Mexico, México & 18 áreas envolvendo onze poços & duas vezes & Transversal & eletrométrico \\
\hline Wambu et al. & 2011 & Nakuru County, Quênia. & vários pontos segundo as fontes & coleta única & Transversal & eletrométrico \\
\hline Azlan et al.* & 2012 & Vale Klang, Malásia & $\begin{array}{l}3 \text { amostras de diferentes locais de doze } \\
\text { estados selecionados da península da } \\
\text { Malásia }\end{array}$ & coleta única & Transversal & eletrométrico \\
\hline Gupta et al.* & 2012 & cidade de Agra, Uttar Pradesh, India & duas fontes & coleta única & Transversal & eletrométrico \\
\hline Lupo et al. & 2012 & regiões da Província de Santa Fé, Argentina & diferentes áreas da província & coleta única & Transversal & eletrométrico \\
\hline Sarmah et al. & 2012 & $\begin{array}{l}\text { Bihpuria de Lakhimpur, Distrito de Assam, } \\
\text { Índia. }\end{array}$ & 12 pontos de coleta & coleta única & Transversal & eletrométrico \\
\hline
\end{tabular}

Nota: *também examinou água engarrafada; **também examinou alimentos; *** técnica espectofotométrica e condutométrica combinadas.

\section{IPABH}




\section{RESULTADOS E DISCUSSÃO}

Após a aplicação dos critérios de inclusão pré-definidos para os títulos e resumos, 113 registros elegíveis foram identificados de um total de 2.416 registros. Trinta e seis artigos foram incluídos para extração dos dados depois da remoção de duplicatas, avaliação do texto na íntegra e busca manual da lista de referências nos artigos incluídos, dos quais 28 estudos foram realizados em localidades das Américas, sendo $24(66,7 \%)$ no Brasil, e os demais distribuídos na Argentina, Canadá, México e Estados Unidos. Cinco foram realizados na Ásia, sendo três na Índia, um no Irã, e um na Malásia. Os demais eram referidos ao Quênia e à Nigéria, pertencentes ao continente africano, e à Itália na Europa. Na Tabela 1 são mostrados os estudos realizados em localidades brasileiras e na Tabela 2 os estudos realizados fora do Brasil.

O fluxo de seleção dos artigos é apresentado na Figura 1.

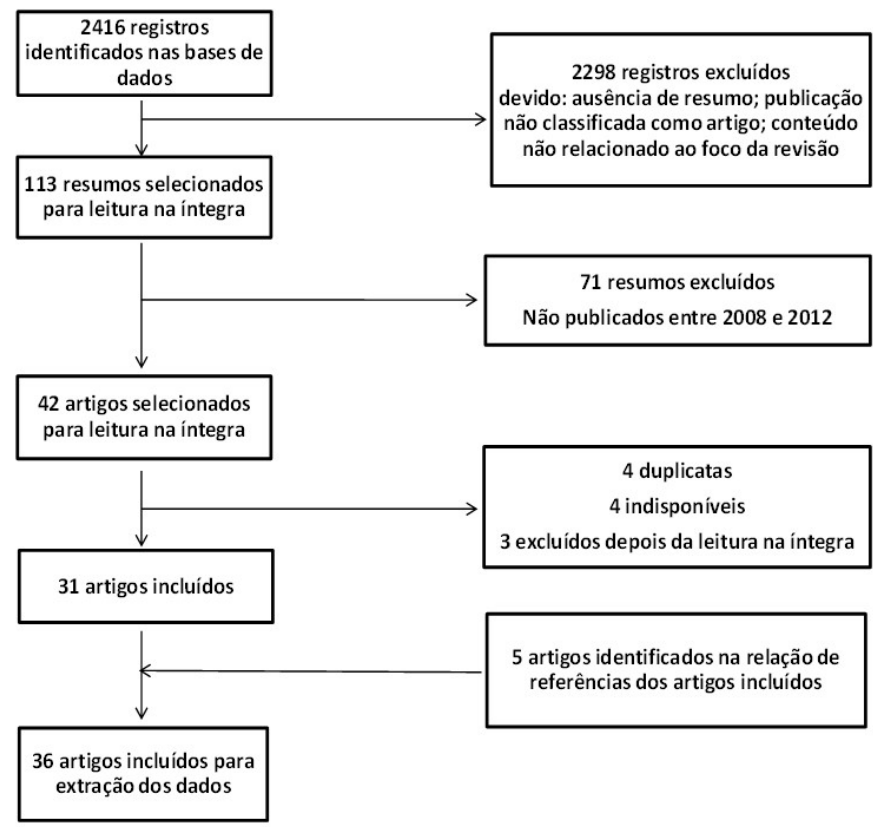

Figura 1. Fluxograma da busca e seleção dos estudos.

No Brasil, a maioria dos estudos concentrou-se na região Sudeste $(\mathrm{N}=13)$, seguido pela região Nordeste $(\mathrm{N}=5)$, região Sul $(\mathrm{N}=4)$, e região Centro-Oeste $(\mathrm{N}=1)$. Uma investigação envolveu as capitais brasileiras. Cabe destacar que um dos estudos incluídos na região Sudeste, envolveu nove localidades da região e uma cidade de fora da região (Tabela 1).

Em relação à distribuição espaço-temporal das amostras, entre os 36 estudos, apenas dois não informaram o tempo da coleta das amostras (Poureslami et al. 2008; Viswanathan, 2009) e três não informaram a distribuição dos pontos de coleta das amostras no território (Poureslami et al., 2008; Scorsafava et al., 2008; Silva et al., 2011).

Quanto à duração temporal, $16(44,4 \%)$ estudos foram considerados transversais e 20 $(55,6 \%)$ longitudinais. Entre os transversais que mencionaram detalhes sobre o tempo, a coleta das amostras ocorreu uma única vez em 10 (27,8\%) estudos (dos quais três brasileiros); e ocorreu em um período entre dois e três meses em $5(13,9 \%)$ estudos. A frequência mensal da coleta das amostras predominou nos estudos longitudinais, sendo três $(8,3 \%)$ com duração entre 5 e 9 meses, nove $(25,0 \%)$ com duração entre 12 e 48 meses, e oito $(22,2 \%)$ com duração de 60 meses ou mais. Em um dos estudos, a duração temporal variou de 36 a 132 meses segundo a localidade (Tabelas 1 e 2). 
Em relação à distribuição espacial das amostras no território, a descrição na maioria dos estudos foi bastante sucinta indicando apenas o número de pontos de coleta (Tabelas 1 e 2). A justificativa geral mencionada foi buscar representar todo o sistema de distribuição (Daré et al. 2009). Entre os estudos que descreveram critérios para a distribuição espacial das amostras, pode-se distinguir aqueles que definiram o número de pontos de coleta conforme o porte populacional (D'Alessandro et al., 2008; Catani et al., 2008; Panizzi e Peres, 2008); e aqueles que definiram os pontos segundo o número de unidades de tratamento ou fontes de abastecimento (Saliba et al., 2009; Silva et al., 2009; Olivati et al., 2011; Moimaz et al. 2012a; 2012b; 2012c). Dois estudos combinaram mais de um critério. Peixoto et al. (2012) adotaram três pontos por unidade de tratamento e um mínimo de cinco pontos de coleta por município com até 50 mil habitantes, e Scorsafava et al. (2011) consideraram, além da densidade populacional, locais estratégicos de baixa pressão do sistema e os registros de distribuição espacial das doenças hídricas. Por analisar dados anuais provenientes de relatórios de qualidade da água exigidos por legislação específica, dois estudos mencionaram documentos técnicos para a obtenção de detalhes sobre a coleta das amostras (Yarmolinsky et al., 2009; Cesa et al., 2011).

A distribuição das técnicas de aferição é apresentada nas Tabelas 1 e 2 . O método para determinação da concentração de fluoreto nas águas mais utilizado foi o eletrométrico (32/36). O método colorimétrico por meio da técnica de SPANDS foi relatado em um estudo (Moraes et al. 2009). Em um artigo foram comparados ambos os métodos (Motter et al. 2011). Akpata et al. (2009) mencionaram técnicas espectofotométrica e condutométrica combinadas. Dois estudos não informaram, pois tinham por base dados anuais provenientes de relatórios de qualidade da água exigidos por legislação específica (Yarmolinsky et al., 2009; Cesa et al., 2011).

Conforme mostra as Tabelas 3 e 4, a classificação dicotômica foi empregada em 27 (75\%) estudos; a tricotômica em cinco (13,9\%) e a multinominal em quatro $(11,1 \%)$. Dezessete $(47,2 \%)$ estudos utilizaram média, desvio-padrão e porcentagem para descrever as estimativas e dezesseis $(44,4 \%)$ usaram média e/ou porcentagem. Apenas dois empregaram mediana (Leivas et al., 2010; Lupo et al., 2012) e um usou coeficiente de variação (D'Alessandro et al., 2008).

Quanto aos vinte e quatro estudos brasileiros, dois deles interpretaram os resultados comparando três critérios diferentes: Panizzi e Peres (2008) utilizaram a Portaria 518/04 (Brasil, 2004), a Portaria 635/75 (Brasil, 1975) e Narvai modificado por Ramires (2006); enquanto Peixoto et al. (2012) se apoiaram na Portaria 635/75, em Ramires et al. (2006) e CECOL/USP (2011). Catani et al. (2008) utilizaram o critério referido por Narvai (2000a). Dezessete estudos relataram apenas um critério, sendo a legislação relativa à potabilidade da água (Portaria 518/04) e à fluoretação (Portaria 635/75), que foram os fundamentos mais mencionados para avaliar os valores observados. Um estudo no estado do Rio de Janeiro (Marmolejo e Coutinho, 2010) utilizou o critério apoiado em Ramires et al. (2006). Dos oito estudos realizados na região Sudeste, todos utilizaram apenas um critério que correspondeu à Portaria 518/04, ou à legislação específica do estado de São Paulo (Resolução SS 250/95), ou ao documento de consenso técnico divulgado pelo Centro Colaborador do Ministério da Saúde em Vigilância da Saúde Bucal da Universidade de São Paulo - CECOL/USP (2011). Dos cinco estudos realizados na região Nordeste relacionados à água de abastecimento, quatro adotaram apenas um critério. Três estudos fundamentaram a interpretação dos valores na Portaria 518/04 e na Portaria 635/75, e um estudo utilizou o critério de Galagan e Vermillion 1957). Na região Sul, apenas um estudo lançou mão de três critérios (Panizzi e Peres, 2008). Os demais utilizaram apenas um critério: Portaria 635/75 (Moraes et al., 2009; Motter et al., 2011); e a Portaria SS 10/99 - SES/RS (Leivas et al., 2010) (Tabela 3).

Dos doze estudos realizados fora do Brasil, oito utilizaram recomendações da 
Organização Mundial da Saúde (OMS) para interpretar a concentração de fluoreto na água (Tabela 4). Os demais empregaram critérios específicos de organismos nacionais, como por exemplo: "U. S. Environmental Protection Agency; U.S. Center for Disease Control and Prevention; Ministry of the Environment and Climate Change in Canada; Kenya Bureau of Standards; Indian Standard Institute."

A atividade de monitoramento foi articulada à vigilância como ação do Estado em 16 estudos $(44,4 \%)$, dos quais 15 eram estudos brasileiros. Essa articulação foi observada por meio de sua menção explícita nos procedimentos de mapeamento da rede de abastecimento e coleta das amostras. Seis estudos fizeram uso de dados de concentração de fluoreto em águas de consumo humano produzidos pelas autoridades sanitárias, sendo um deles no Canadá (Yarmolinsky et al., 2009) e os demais no Brasil (Panizzi e Peres, 2008; Scorsafava et al., 2008; 2011; Cesa et al., 2011; Motter et al., 2011). Entre os estudos não brasileiros, apenas três interpretaram os resultados com base em agências governamentais do próprio país responsável pela aprovação de padrões de referência e normas (Quock e Chan, 2010; Wambu et al., 2011; Sarmah et al. 2012).

A identificação de possíveis fatores associados aos resultados encontrados sobre a concentração de fluoreto foi escassa, e a maioria dos estudos comentou o seu significado do ponto de vista do benefício ou do risco para a saúde da dentição. Um aspecto comum em muitos estudos é a descontinuidade da medida caracterizada por valores de concentração em desacordo com a legislação e abaixo do indicado para prevenção da cárie.

A operação e manutenção adequadas dos equipamentos utilizados no processo de fluoretação são fundamentais para garantir o fornecimento contínuo da água com concentrações ótimas de íons fluoreto. Entre os possíveis fatores associados levantados pelos estudos destacaram-se: padrão geológico do solo (Silva et al., 2009; Sampaio et al., 2010; D'Alessandro et al., 2008; Akpata et al., 2009; Viswanathan, 2009; Wambu et al., 2011; Lupo et al., 2012); áreas rurais em comparação com urbanas (Yarmolinsky et al., 2009; Olivati et al., 2011); tipo de manancial, porte do sistema, concessionária, o nível de desenvolvimento humano municipal (Daré et al., 2009); tipo de sistema de abastecimento: estação de tratamento; estação de tratamento e poço tubular profundo; poço tubular profundo (Silva et al., 2011); abastecimento por sistemas de poços artesianos, o que dificultaria, sobremaneira, o controle operacional do método (Bellé et al., 2009); problemas no equipamento hidráulico ou em variações no fluxo de água (vazão) ao longo da rede de distribuição da cidade, velocidade de esvaziamento dos reservatórios distribuídos que não estão interligados entre si (Marmolejo e Coutinho, 2010); dificuldades operacionais e interpretação da legislação (Panizzi e Peres, 2008; Moraes et al., 2009; Saliba et al., 2009); o tipo de técnica analítica (Motter et al., 2011). Aventou-se ainda a presença natural de fluoreto nas águas de alguns municípios (Scorsafava et al., 2011) e sugeriu-se que quanto menor o número de fontes de água no município, melhor o controle (Moimaz et al., 2012b).

Além dos fatores ligados à área geográfica, à concessionária, ao tipo de sistema e à técnica analítica, descritos nos estudos revisados, problemas com fornecimento do composto utilizado também podem alterar os resultados relativos à concentração de fluoreto (Maia et al., 2003).

Embora muitos estudos na forma de artigos tenham sido divulgados sobre monitoramento da concentração de fluoreto nas águas de abastecimento, este trabalho é a primeira revisão sistemática oferecendo um balanço sobre a produção do conhecimento publicado na literatura científica na forma de artigos em revistas especializadas. Embora restrita ao período de 2008 a 2012, artigos provenientes dos quatro principais continentes foram identificados e algumas importantes características teórico-metodológicas emergiram da análise.

Rev. Ambient. Água vol. 11 n. 4 Taubaté - Oct. / Dec. 2016 


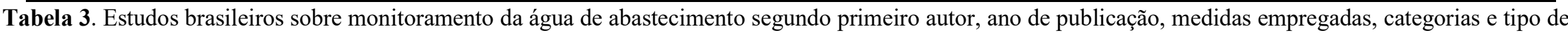
classificação e critério de avaliação.

\begin{tabular}{|c|c|c|c|c|c|}
\hline $1^{\circ}$ Autor & Ano & Medidas & Categorias de classificação & Tipo de classificação & Critério de avaliação \\
\hline Alves et al. & 2008 & Média & Conformidade ao padrão (acima/abaixo) & dicotômico & Portaria $635 / 75$ \\
\hline Catani et al. & 2008 & Média (d.p.) e porcentagem & Conformidade ao padrão (aceitável/inaceitável) & dicotômico & Narvai (2000) \\
\hline Fernandes-Jr et al. & 2008 & Média (d.p.) e porcentagem & Adequado/inadequado e acima/abaixo & dicotômico & Portaria 518/04 Portaria 635/75 \\
\hline Panizzi e Peres & 2008 & Média (d.p.) e porcentagem & Adequado/inadequado e acima/abaixo & dicotômico & $\begin{array}{l}\text { Portaria } 518 / 04 \text {, Portaria } 635 / 75 \text {, Narvai } \\
\text { modificado por Ramires (2004) }\end{array}$ \\
\hline Scorsafava et al. & 2008 & Porcentagem & Adequado/inadequado e acima/abaixo & dicotômico & Resolução SS 250/95 - SP \\
\hline Bellé et al. & 2009 & Média e porcentagem & Conformidade ao padrão (aceitável/inaceitável) & dicotômico & Portaria 635/75 \\
\hline Daré et al. & 2009 & Média (d.p.) e porcentagem & Adequado/inadequado e acima/abaixo & dicotômico & Portaria 518/04 Portaria 635/75 \\
\hline Moraes et al. & 2009 & Média e porcentagem & Conformidade ao padrão (aceitável/inaceitável) & dicotômico & Portaria $635 / 75$ \\
\hline Saliba et al. & 2009 & Porcentagem & Adequado/inadequado e acima/abaixo & dicotômico & Portaria 518/04 Portaria 635/75 \\
\hline Silva et al. & 2009 & Média & $<0,31 \mathrm{mg} / \mathrm{L} ; 0,31-0,59 ; 0,60-0,80 ;>0,80 \mathrm{mg} / \mathrm{L}$ & multinominal & Galagan e Vermillion 1957 \\
\hline Carmo et al. & 2010 & Média e porcentagem & Conformidade ao padrão (aceitável/inaceitável) & dicotômico & Portaria $635 / 75$ \\
\hline Leivas et al. & 2010 & Média (d.p.) mediana e porcentagem & Adequado/inadequado e acima/abaixo & dicotômico & Portaria SS 10/99 - RS \\
\hline Marmolejo et al. & 2010 & Média & Conformidade ao padrão (acima/abaixo) & dicotômico & Ramires et al. (2006) \\
\hline Sampaio et al. & 2010 & Média (d.p.) e porcentagem & Adequado/inadequado e acima/abaixo & dicotômico & Portaria 518/04 Portaria 635/75 \\
\hline Castro et al.* & 2011 & Média (d.p.) e porcentagem & Conformidade ao padrão ( acima /abaixo) & dicotômico & Portaria 518/04 \\
\hline Cesa et al. & 2011 & média e porcentagem & Conformidade ao padrão (Adequado/inadequado) & dicotômico & Portaria 518/04 Portaria 635/75 \\
\hline Motter et al. & 2011 & Média (d.p.) e porcentagem & Conformidade ao padrão (Adequado/inadequado) & dicotômico & Portaria $635 / 75$ \\
\hline Olivati et al. & 2011 & Média e porcentagem & Conformidade ao padrão (Adequado/inadequado) & dicotômico & Portaria $635 / 75$ \\
\hline Scorsafava et al. & 2011 & Média e porcentagem & Conformidade ao padrão (aceitável/inaceitável) & dicotômico & Resolução SS 250/95 - SP \\
\hline Silva et al. & 2011 & Média e porcentagem & Conformidade ao padrão (acima/abaixo) & dicotômico & Resolução SS 250/95 - SP \\
\hline Moimaz et al & 2012 & Média (d.p) e porcentagem & $\begin{array}{l}0,00-0,44 ; 0,45-0,54 ; 0,55-0,84 ; 0,85-1,14 ;>1,14 \mathrm{mg} / \mathrm{L} \text {. } \\
\text { Combinação risco-benefício }\end{array}$ & multinominal & CECOL/USP (2011) \\
\hline Moimaz et al & 2012 & Média (d.p.) e porcentagem & $\begin{array}{l}0,00-0,44 ; 0,45-0,54 ; 0,55-0,84 ; 0,85-1,14 ; 1,15-1,44 ;>1,44 \\
\text { mg/L. Combinação risco-benefício }\end{array}$ & multinominal & CECOL/USP (2011) \\
\hline Moimaz et al. & 2012 & Média (d.p) e porcentagem & $<0,6 \mathrm{mg} / \mathrm{L} ; 0,6-0,8 \mathrm{mg} / \mathrm{L} ;>0,8 \mathrm{mg} / \mathrm{L}$ & tricotômico & Resolução SS 250/95 - SP \\
\hline Peixoto et al. & 2012 & Média (d.p.) e porcentagem & Conformidade ao padrão + Combinação risco-benefício & dicotômico/multinominal & $\begin{array}{l}\text { Portaria 635/75, Ramires et al. 2006; } \\
\text { CECOL/USP } 2011\end{array}$ \\
\hline
\end{tabular}

IPABH 
Tabela 4. Estudos não brasileiros sobre monitoramento da água de abastecimento segundo primeiro autor, ano de publicação, medidas empregadas, categorias e tipo de classificação e critério de avaliação.

\begin{tabular}{|c|c|c|c|c|c|}
\hline $1^{\circ}$ Autor & Ano & Medidas & Categorias de classificação & Tipo de classificação & Critério de avaliação \\
\hline D'Alessandro et al. & 2008 & Min-Max, Percentis, CV, porcentagem & $<0,5 \mathrm{mg} / \mathrm{L} ; 0,5-1,5 \mathrm{mg} / \mathrm{L} ;>1,5 \mathrm{mg} / \mathrm{L}$ & Tricotômica & OMS \\
\hline Poureslami et al. ${ }^{* *}$ & 2008 & Média (d.p.) e porcentagem. & Conformidade ao padrão (acima/abaixo) & Dicotômica & $\mathrm{CDC}$ \\
\hline Akpata et al. & 2009 & Média (d.p.) e porcentagem. & $<0,3 ; 0,3-0,6 ;>0,6 \mathrm{mg} / \mathrm{L}$. Conforme $1,5 \mathrm{mg} / \mathrm{L}$ & Tricotômica & autores; OMS \\
\hline Viswanathan & 2009 & Média (d.p.) e porcentagem. & Conformidade ao padrão (acima/abaixo) & Dicotômica & OMS \\
\hline Yarmolinsky et al. & 2009 & Média & $\begin{array}{l}<0,5 \mathrm{mg} / \mathrm{L} ; 0,5-0,8 \mathrm{mg} / \mathrm{L} ;>0,8 \mathrm{mg} / \mathrm{L} \text {. Conforme } \\
0,7 \pm 0,1 \mathrm{mg} / \mathrm{L}\end{array}$ & Tricotômica & $\begin{array}{l}\text { MOE; Health } \\
\text { Canada }\end{array}$ \\
\hline Quock \& Chan & 2010 & Média (d.p.), IC95\%, mediana & $<0,7 \mathrm{mg} / \mathrm{L} ; 0,7-1,2 \mathrm{mg} / \mathrm{L} ;>1,2 \mathrm{mg} / \mathrm{L}$ & Tricotômica & EPA \\
\hline Chacón et el. & 2011 & média (d.p.) e IC95\%, porcentagem & Conf. ao padrão (acima/abaixo) & Dicotômica & OMS \\
\hline Wambu et al. & 2011 & Média (dp) e porcentagem. & Conf. ao padrão (acima/abaixo) & Dicotômica & KBS \\
\hline Azlan et al.* & 2012 & Média & Conf. ao padrão (acima/abaixo) & Dicotômica & OMS \\
\hline Gupta et al.* & 2012 & Média & Conf. ao padrão (acima/abaixo) & Dicotômica & OMS \\
\hline Lupo et al. & 2012 & Mediana & Conf. ao padrão (acima/abaixo) & Dicotômica & OMS \\
\hline Sarmah et al. & 2012 & Média & Conf. ao padrão (acima/abaixo) & Dicotômica & ISI, OMS \\
\hline
\end{tabular}

Nota. *também examinou água engarrafada; **também examinou alimentos. Siglas. OMS: Organização Mundial da Saúde; CDC: U.S. Center for Disease Control and Prevention; MOE: Ministry of the Environment; EPA: Environmental Protection Agency; KBS: Kenya Bureau of Standards; ISI: Indian Standard Institute. 
Os resultados mostraram a grande variabilidade dos estudos quanto ao desenho e aos procedimentos metodológicos. A maior parte dos estudos identificados foi realizada em localidades brasileiras. Tal predomínio pode ser atribuído ao interesse dos pesquisadores brasileiros em relação à concentração do fluoreto na água desde que a fluoretação foi adotada como uma política oficial no país. O maior número de estudos na região sudeste pode estar relacionado à maior quantidade de unidades produtoras de ciência e tecnologia existentes na região quando comparada às demais regiões do país (Brasil, 2010). Entre os estudos estrangeiros, foi elevado o número de estudos no continente asiático, fato que pode estar associado à presença de muitas áreas com fluoreto de ocorrência natural em níveis acima do seguro para a saúde humana (Australian Government, 2007).

Embora a maioria dos estudos $(88,9 \%)$ tenha oferecido alguma informação sobre a distribuição espaço-temporal das amostras, em poucos trabalhos foi possível inferir a população coberta pela fonte de abastecimento pesquisada. A informação sobre a distribuição espaço-temporal das amostras é muito importante para avaliar o grau com que as amostras representam o universo relativo à água distribuída à população e para possibilitar a interpretação dos resultados tanto do ponto de vista da população atingida quanto das características da água em relação ao parâmetro sob análise. Sua definição deve ser compatível com a finalidade de cada estudo. Por exemplo, em relação à frequência de coleta das amostras, as atividades de monitoramento em áreas de ocorrência natural do fluoreto não seguem as mesmas exigências de atividades de monitoramento em áreas onde a concentração de fluoreto é artificialmente ajustada para fins de prevenção de cárie dentária, em que a maioria dos estudos adotou a frequência mensal. Em relação à distribuição espacial, são poucos os estudos que descrevem detalhadamente os procedimentos adotados para assegurar que os pontos de coleta representem a água de abastecimento oferecida à população. Entre os critérios descritos para a definição dos pontos, destacaram-se o porte populacional e o número de unidades de tratamento ou fontes de abastecimento. Em documento de consenso técnico de especialistas, recomenda-se a obtenção de pelo menos três amostras por mês de cada sistema ou fonte de abastecimento, obtidas no mesmo dia em diferentes pontos do território abastecido pelo respectivo sistema, de modo a reunir pelo menos 36 amostras de um determinado sistema no período de um ano (CECOL, 2011). Um dado adicional relevante é indicar a população coberta por cada sistema ou fonte de abastecimento, um aspecto negligenciado na maioria dos estudos. Quanto à duração temporal, apenas 55,6\% dos estudos foram considerados com desenho longitudinal (coleta durante quatro meses ou mais), o que limita a inferência sobre o alcance dos resultados.

O método eletrométrico foi empregado na maioria das publicações $(88,9 \%)$ corroborando evidências que sublinham as vantagens da técnica do eletrodo íon-seletivo comparada a outras técnicas analíticas (Freitas et al., 2003; Bratovcic et al., 2009).

Quanto às medidas utilizadas para a apresentação dos resultados, predominou o uso do valor médio, do desvio-padrão e da porcentagem. Entretanto, em 44,4\% dos estudos nenhuma medida de dispersão ou de variação foi associada ao valor médio, o que pode dificultar a interpretação dos resultados. Em relação ao tipo de julgamento adotado, a maioria dos estudos (75\%) adotou a classificação dicotômica, do tipo adequado/inadequado e/ou acima/abaixo comparado ao padrão de referência selecionado, sem levar em consideração que o fluoreto presente na água é um fator de proteção ou de risco à dentição dependendo da sua concentração e que valores inadequados deveriam ser discriminados conforme o binômio risco/proteção.

Para interpretar os resultados, embora a maioria dos estudos brasileiros $(70,8 \%)$ tenha lançado mão de apenas um critério de referência, cabe destacar que os critérios não foram os mesmos, sendo empregados até oito diferentes métodos de julgamento. Essa diversidade de critérios levou a que os especialistas brasileiros produzissem um consenso técnico para classificação de águas de abastecimento público segundo o teor de flúor a fim de elevar a 
comparabilidade entre os resultados observados pelos diferentes estudos (CECOL, 2011). Entre os estudos estrangeiros, cabe destacar que oito utilizaram as recomendações da OMS para interpretar a concentração de fluoreto na água, sendo que esse critério está voltado apenas para avaliar o potencial risco à dentição e a saúde humana, sem considerar o efeito de proteção à cárie propiciado por águas com teores entre $0,5 \mathrm{mgF} / \mathrm{L}$ preconizado em Hong Kong na Ásia, e 1,0 mgF/L recentemente aprovado no Canadá, duas áreas geográficas de condições climáticas distintas (Frazão et al., 2011).

Menos da metade dos estudos $(44,4 \%)$ esteve articulada à vigilância como ação do Estado, e um número ainda menor levantou hipóteses sobre possíveis fatores associados aos resultados encontrados, o que revela a necessidade de maior aproximação e interação entre os pesquisadores e os órgãos de controle da qualidade da água. A descontinuidade da medida caracterizada por valores de concentração em desacordo com a legislação e abaixo do indicado para prevenção da cárie foi um achado comum, reforçando as advertências de vários pesquisadores sobre a necessidade da expansão dos sistemas de vigilância da fluoretação da água de abastecimento público. Embora não exposta ao risco de fluorose dentária, a população estaria sendo privada do máximo benefício proporcionado pelo fluoreto em nível adequado na água.

\section{CONCLUSÃO}

Os principais fatores intervenientes no estudo de fluoretos em águas de abastecimento público podem ser agrupados em: aspectos ligados à área geográfica, incluindo-se o padrão geológico do solo, o tipo de manancial, o tipo de área (urbana ou rural), e o grau de desenvolvimento humano; aspectos concernentes à concessionária, ao tipo de sistema e sua operação, incluindo características da rede de distribuição; e aspectos ligados à técnica analítica. A variabilidade dos estudos quanto ao desenho e aos procedimentos metodológicos foi elevada. Embora a maioria dos estudos tenha oferecido alguma informação sobre a distribuição espaço-temporal das amostras, em poucos trabalhos foi possível inferir a população coberta pela fonte de abastecimento investigada. Quase metade dos estudos foi do tipo transversal e nenhuma medida de dispersão ou de variação foi associada ao valor médio. Predominou o uso do método eletrométrico. A maioria dos estudos adotou a classificação dicotômica, do tipo adequado/inadequado e/ou acima/abaixo. Menos da metade dos estudos esteve articulada à vigilância como ação do Estado, e um número ainda menor levantou hipóteses sobre possíveis fatores associados aos resultados encontrados confirmando que há um espaço importante para melhorar os procedimentos metodológicos em futuros estudos. Além disso, a distância entre os resultados dessas iniciativas e o uso das informações pelas autoridades de saúde pública pode ser diminuído, e a interação entre os pesquisadores e os órgãos de controle da qualidade da água deveria ser incrementada.

\section{REFERÊNCIAS}

AKPATA, E. S. et al. Geographical mapping of fluoride levels in drinking water sources in Nigeria. African Health Science, v. 9, n. 4, p. 227 - 233, 2009.

AUSTRALIAN GOVERNMENT. National Health and Medical Research Council. A systematic review of the efficacy and safety of fluoridation. Part A: review of methodology and results. Canberra, 2007. p. 17-22.

BARROS, E. R. C. et al. Análise crítica da fluoretação de águas no município de Porto Alegre/RS. RGO, v. 38, n. 4, p. 247-54, 1990. 
BELLÉ, B. L. L. et al. Análise da fluoretação da água de abastecimento público da zona urbana do município de Campo Grande (MS). Ciência e Saúde Coletiva, v. 14, n. 4, p. 1261-1266, 2009.

BRASIL. Ministério da Saúde. Portaria no 635/ BSB, de 25 de dezembro de1975. Aprova as normas e padrões sobre a fluoretação da água dos sistemas públicos de abastecimento destinada ao consumo humano. Diário Oficial [da] União, Brasília, DF, 30 jan. 1976.

BRASIL. Ministério da Saúde. Portaria $n^{\circ}$. 518, de 25 de março de 2004. Estabelece os procedimentos e responsabilidades relativas ao controle e vigilância da qualidade da água para o consumo humano e seu padrão de potabilidade, e dá outras providências. Diário Oficial [da] União, Brasília, DF, p. 266-70, 26 mar. 2004. Seção 1.

BRASIL. Ministério da Educação. Coordenação de Aperfeiçoamento de Pessoal de Nível Superior. Plano Nacional de Pós-Graduação - PNPG 2011-2020. Brasília, DF: CAPES, 2010.

BRATOVCIC, A.; ODOBASIC, A.; CATIC, S. The Advantages of the Use of Ion-Selective Potentiometry in Relation to UV/VIS Spectroscopy. Agriculturae Conspectus Scientificus, v. 74, n. 3, p. 139-41, 2009.

CARMO, C. D. S. et al. Avaliação da fluoretação da água do sistema de abastecimento público na Ilha de São Luís, Maranhão, Brasil. Ciência e Saúde Coletiva, v. 15, supl. 1, p. 1835-1840, 2010.

CATANI, D. B. et al. Dez anos de acompanhamento do heterocontrole da fluoretação da água feito por municípios brasileiros, Brasil, 1996-2006. RGO, v. 56, n. 2, p. 151-155, 2008.

CENTRO COLABORADOR DO MINISTÉRIO DA SAÚDE EM VIGILÂNCIA DA SAÚDE BUCAL - CECOL/USP. Consenso técnico sobre classificação de águas de abastecimento público segundo teor de flúor. São Paulo: Faculdade de Saúde Pública da Universidade de São Paulo; 2011. Disponível em: http://www.cecol.fsp.br/dcms/ fck/file/SeminarioVigifluor2011/CECOL/USP. ClassificacaoAguasSegundoteordefluor DocumentoConsensoTecnico2011(2).

CESA, K. et al. A vigilância da fluoretação de águas nas capitais brasileiras. Epidemiologia e Serviços de Saúde, v. 20, n. 4, p. 547-555, 2011. http://dx.doi.org/10.5123/S167949742011000400014

CHAN, J. T. et al. Fluctuations in tap water fluoride levels: a potential problem for practitioners. Texas Dental Journal, v. 110, n. 2, p. 17-20, 1993.

D'ALESSANDRO, W. et al. Survey on fluoride, bromide and chloride contents in public drinking water supplies in Sicily (Italy). Environmental Monitoring and Assessment, v. 145, n. 1-3, p. 303-313, 2008. http://dx.doi.org/10.1007/s10661-007-0039-y

DARÉ, F. et al. Avaliação do processo de fluoretação nos sistemas de abastecimento de água da região de Araçatuba, São Paulo. Engenharia Sanitária e Ambiental, v. 14, n. 2, p. 173-182, 2009.

ENCONTRO NACIONAL DE ADMINISTRADORES E TÉCNICOS DO SERVIÇO PÚBLICO ODONTOLÓGICO - ENATESPO. 1998. Documento Final. Fortaleza, 1998.

FRAZÃO, P. et al. Drinking water quality and fluoride concentration. Revista de Saúde Pública, v. 45, n. 5, p. 964-73, 2011. 
FREITAS, V. P. S. et al. Estudo comparativo de soluções-tampão para determinação de fluoreto em águas para diálise. Revista do Instituto Adolfo Lutz, v. 62, n. 3, p. 207-12, 2003.

GALAGAN, D. J.; VERMILLION, J. R. Determining optimum fluoride concentrations. Public Health Reports, v. 72, n. 6, p. 491-3, 1957.

LEIVAS, L. L. et al. Heterocontrole da fluoretação das águas de abastecimento público do Município de Canoas/RS. Stomatos, v. 16, n. 30, 2010.

LUPO, M. et al. Determination of water fluoride concentration and the influence of the geographic coordinate system and time. Water, Air, and Soil Pollution, v. 223, n. 8, p. 5221-5225, 2012. http://dx.doi.org/10.1007/s11270-012-1273-7

MAIA, L. C. et al. Controle Operacional da fluoretação da água de Niterói, Rio de Janeiro, Brasil. Cadernos de Saúde Pública, v. 19, n. 1, p. 61-7, 2003. http://dx.doi. org/10.1590/S0102-311X2003000100007

MARMOLEJO, L. D. M. G.; COUTINHO, T. C. L. Heterocontrole da fluoretação da água de abastecimento público em Niterói, RJ, Brasil, no período de novembro de 2008 a março de 2009. Revista Fluminense de Odontologia, v. 16, n. 33, p. 34-39, 2010.

MOIMAZ, S. A. S. et al. External control of the public water supply in 29 Brazilian cities. Brazilian Oral Research, v. 26, n. 1, p. 12-18, 2012a. http://dx.doi.org/10.1590/S180683242012000100003

MOIMAZ, S. A. S. et. al. Fluoride Concentration in Public Water Supply: 72 Months of Analysis. Brazilian Dental Journal, v. 23, n. 4, p. 451-456, 2012b. http://dx.doi.org/ 10.1590/S0103-64402012000400024

MOIMAZ, S. A. S. et. al. Fluoretação das águas de Abastecimento Público no Município de Araçatuba/SP. Revista de Odontologia de Araçatuba, v. 33, n. 1, p. 54-60, 2012c.

MORAES, J. E. et al. Determinação do índice de fluoreto em águas de abastecimento público em municípios da região centro-sul do Paraná. Ambiência, v. 5, n. 2, p. 233-246, 2009.

MOTTER, J. et. al. Análise da concentração de flúor na água em Curitiba, Brasil: comparação entre técnicas. Revista Panamericana de Saúde Pública, v. 29, n. 2, p. 120-125, 2011.

NARVAI, P. C. Cárie dentária e flúor: uma relação do século XX. Ciência e Saúde Coletiva, v. 5, p. 381-92, 2000a. http://dx.doi.org/10.1590/S1413-81232000000200011

NARVAI, P. C. Fluoretação da água: heterocontrole no município de São Paulo no período de 1990-1999. Revista Brasileira de Odontologia em Saúde Coletiva, v. 2, n. 2, p. 50 $56,2000 \mathrm{~b}$.

OLIVATI, F.N. et. al. Qualidade da fluoretação da água de Capão Bonito, SP, Brasil, avaliada pelos controles operacional e heterocontrole. Revista Odonto Ciência, v. 26, n. 4, p. 285-290, 2011.

PANIZZI, M.; PERES, M. A. Dez anos de heterocontrole da fluoretação de águas em Chapecó, Estado de Santa Catarina, Brasil. Cadernos de Saúde Pública, v. 24, n. 9, p. 2021-2031, 2008. http://dx.doi.org/10.1590/S0102-311X2008000900008 
PEIXOTO, D. F. et at. Heterocontrole da fluoretação da água de abastecimento público do município de Jaguaribara, Ceará, Brasil. Revista Brasileira de Promoção da Saúde, v. 25, n. 3, p. 271-277, 2012.

POURESLAMI, H. R. et al. Fluoride in food and water consumed in Koohbanan (Kuh-e Banan), Iran. Fluoride, v. 41, n. 3, p. 216-219, 2008.

QUOCK, R. L.; CHAN, J. T. Weekly monitoring of the water fluoride content in a fluoridated metropolitan city: results after 1 year. Texas Dental Journal, v. 127, n. 7, p. 665-71, 2010.

RAMIRES, I. et. al. Heterocontrole da fluoretação da água de abastecimento público em Bauru, SP, Brasil. Revista de Saúde Pública, v. 40, n. 5, p. 883-9, 2006. http://dx.doi.org/10.1590/S0034-89102006005000005

SALIBA, N. A. et al. Vigilância do teor de flúor nas águas de abastecimento público de municípios do noroeste paulista, Brasil: 36 meses de análise. Revista Odonto Ciência, v. 24, n. 4 , p. 372-376, 2009.

SAMPAIO, F.C. et al. Natural Fluoride Levels in the Drinking Water, Water Fluoridation and Estimated Risk of Dental Fluorosis in a Tropical Region of Brazil. Oral Health Preventive Dentistry, v. 8, p. 71-75, 2010.

SARMAH, S. P. et al. Evaluation of drinking water quality in Bihpuria area of Lakhimpur District, Assam, India. Research Journal of Pharmaceutical, Biological and Chemical Sciences, v. 3, p. 1030-1036, 2012.

SCHNEIDER FILHO, D. A. et al. Fluoretação da água: como fazer a vigilância sanitária? Cadernos de Saúde Bucal, Rio de Janeiro: Cedros, 1992.

SCORSAFAVA, M. A. et al. Avaliação da qualidade da água de abastecimento no período 2007- 2009. Revista do Instituto Adolfo Lutz, v. 70, n. 3, p. 395-403, 2011.

SCORSAFAVA, M. A. et al. Controle físico-químico da qualidade da água para consumo humano na região da grande São Paulo. Boletim do Instituto Adolfo Lutz, v. 18, n. 1/2, p. 44, 2008.

SILVA, J. S. et al. Natural fluoride levels from public water supplies in Piauí State, Brazil. Ciência e Saúde Coletiva, v. 14, n. 6, p. 2215-2220, 2009. http://dx.doi.org/ $10.1590 /$ S1413-81232009000600030

SILVA, R. A. et al. Monitoramento da concentração de íons fluoreto na água destinada ao consumo humano proveniente de um município com diferentes sistemas de abastecimento público. Revista do Instituto Adolfo Lutz, v. 70, n. 2, p. 220-224, 2011.

TRICCO, A. C. et al. The art and science of knowledge synthesis. Journal of Clinical Epidemiology, v. 64, n. 1, p. 11-20, 2011. http://dx.doi.org/10.1016/ j.jclinepi.2009.11.007

VASCONCELLOS, M. C. C. Prevalência de cárie dentária em escolares de 7 a 12 anos de idade, na cidade de Araraquara, SP (Brasil), 1979. Revista de Saúde Pública, v. 16, p. 317-28, 1982. http://dx.doi.org/10.1590/S0034-89101982000600002

VISWANATHAN, G. et al. Mapping of fluoride endemic areas and assessment of fluoride exposure. Science of the Total Environment, v. 407, p. 1579-1587, 2009. http://dx.doi.org/10.1016/j.scitotenv.2008.10.020 
YARMOLINSKY, J. et al. Variation in urban and rural water fluoride levels in Ontario. Journal of Canadian Dental Association, v. 75, n. 10, p. 707, 2009.

WALDMAN, E. Vigilância em Saúde Pública. São Paulo: Faculdade de Saúde Pública da Universidade de São Paulo, 1998.

WAMBU, E. W. et al. High fluoride water in the Gilgil area of Nakuru County, Kenya. Fluoride, v. 44, n. 1, p. 37-41, 2011.

WORLD HEALTH ORGANIZATION - WHO. Guidelines for drinking-water quality. 4. edition. Geneva, 2011. 